\title{
Hydrodynamic Hull Form Design Using an Optimization Technique
}

\author{
Dong-Woo Park ${ }^{1}$ and Hee-Jong Choi ${ }^{2 *}$ \\ ${ }^{1}$ Maritime Research Institute, Hyundai Heavy Industries, Ulsan 682-792, Korea \\ ${ }^{2}$ Department of Naval Architecture and Ocean Engineering, Chonnam National University, Yeosu 550-749, Korea
}

(Manuscript Received December 6 2012; Revised January 15, 2013; Accepted February 20, 2013)

\begin{abstract}
A design procedure for a ship with minimum resistance had been developed using a numerical optimization method called SQP (Sequential Quadratic Programming) combined with computational fluid dynamics (CFD) technique. The frictional resistance coefficient was estimated by the ITTC 1957 model-ship correlation line formula and the wave-making resistance coefficient was evaluated by the potential-flow panel method with the nonlinear free surface boundary conditions. The geometry of the hull surface was represented and modified by B-spline surface modeling technique during the optimization process. The Series $60\left(C_{B}=0.60\right)$ hull was selected as a parent hull to obtain an optimized hull that produces minimum resistance. The models of the parent and optimized hull forms were tested at calm water condition in order to demonstrate the validity of the proposed methodolgy.
\end{abstract}

Keywords: Hull optimization, Minimum resistance, Ranking source panel method, SQP(Sequential Quadratic Programming), B-spline surface modeling

\section{Introduction}

The resistance of the ship is determined by hydrodynamic forces, which strongly depend on the shape of the ship. Optimizing the hull form from the hydrodynamic point of view is a complicated problem; not only by the many constraints imposed by practical demands but also by the influence of ambient conditions. Minimum operating fuel costs would be achieved by designing the hull for best average performance over a range of conditions. This is ,however, rarely feasible because of the current difficulties to quantify the effect of all parameters involved and because of the more trivial reason of the severe time constraints that are the rule in practical ship design [9].

\footnotetext{
*Corresponding author. Tel.: +82-61-659-7154, Fax.: +82-61-659-7159,

E-mail address: choihj@jnu.ac.kr

Copyright $\odot$ KSOE 2012.
}

With the development of the CFD techniques and the improvement of computer performance, more comprehensive research has been recently carried out from viewpoint of the resistance reduction and interest in the prediction of the optimized hull form satisfying the design constraints has been increasing $[2,6,11]$.

Although some of these researches have provided some encouraging results, there are still a number of significant difficulties: selection of an appropriate objective function, choice of optimization scheme, geometric representation of hull surface and choice of a practical, robust CFD tool to evaluate the objective function and the large computational cost to estimate the objective function through the repeated computations during the entire optimization process.

Recently, many interesting papers on hull form optimization in terms of resistance have been published. Hino et. al. [3] and Tahara et. al. [12, 13] 
developed a ship optimization method using the Navier-Stokes solver and SQP algorithm and performed a hull optimization for a Model 5415 hull. Peri et. al. [8] presented several new optimized bulb shapes for a tanker with the aid of the potential flow solver and three different optimization algorithms. Markov et. al. [7] performed the hull form optimization for the Series 60 hull $\left(\mathrm{C}_{\mathrm{B}}=0.60\right)$ and the HTC (Hamburg Test Case) container carrier using the potential flow solver(the higher-order Rankine source panel method) to evaluate the wave-making resistance and DFP (unconstrained DavidsonFletcher-Powell) as an optimization method and a B-spline patch to approximate the hull surface during the optimization process. Zhang $[15,16]$ performed the hull form optimization using the Ranking source method to achieve the wave-making resistance as the objective function focusing on the optimization of the bow-body shape. In the present study, an hull form optimization algorithm was proposed from viewpoint of the resistance reduction. To achieve the resistance of the ship the wavemaking resistance was computed using the potential flow solver in which the nonlinear free surface boundary conditions and the trim and sinkage of a ship were fully taken into account and the friction resistance is estimated using the ITTC 1957 modelship correlation line formula. During the optimization process the geometry of the hull surface was represented and modified by the B-spline surface modeling technique.

Numerical computations were performed to investigate the validity of the proposed algorithm for the Series 60 hull $\left(\mathrm{C}_{\mathrm{B}}=0.60\right)$ and the parent hull and the optimized hull were manufactured and tested in the towing tank which was installed in South Korea's Pusan National University and the numerical results were compared with the experimental data.

\section{Optimization Program}

\subsection{General Optimization Problem}

The general optimization problem may be expressed in the following form:

Minimize :

$$
f(x)
$$

$m_{e}$ the number of equality constraint functions.
Subjected to:

$g_{j}(x)=0, j=1, \cdots, m_{e}$

$g_{j}(x) \leq 0, j=m_{e}+1, \cdots, m$

$x_{l} \leq x \leq x_{u}$

where $f$ is an objective function, $g$ constraint functions, $x$ the vector of the design variables, $x_{l}$ and $x_{u}$ lower and upper limit of $x, m$ the number of constraint functions and $m_{e}$ the number of equality constraint functions.

\subsection{Nonlinear Programming Algorithm}

In the present study, Eqs. (1), (2) and (3) are solved by sequential quadratic programming algorithm, in which the equations are approximated in quadratic form:

Minimize :

$\frac{1}{2} d^{T} B d+\nabla f(x)^{T} d$

Subjected to:

$\nabla g_{j}(x)^{T} d+g_{j}(x)=0, \quad j=1, \cdots, m_{e}$

$\nabla g_{j}(x)^{T} d+g_{j}(x) \geq 0, \quad j=m_{e}+1, \cdots, m$

where $d$ is search direction vector and $B$ approximate Hessian matrix of the Lagrangian. During the optimization process optimum $d$ is determined and $x$ is updated by $x^{n+1}=x^{n}+d$ in each iteration [14].

\section{Evaluation of an Objective Function}

Define a Cartesian coordinates system fixed on the ship which translates with a constant speed $U$. The $\mathrm{x}$-axis points downstream and the z-axis upwards. In this frame of reference the vessel is stationary experiencing an incoming uniform stream $U$ along the positive $\mathrm{x}$-direction.

The fluid is assumed incompressible, inviscid and irrotational flow, governed by the velocity potential $\phi$ subject to Laplace's equation in the fluid domain.

$\nabla^{2} \phi=0$ 
Over the wetted part of the hull surface the velocity potential must satisfy the hull boundary condition of no flow normal to the surface.

$\phi_{n}=0$

where $n$ is the unit normal vector.

The radiation condition must be satisfied

$\nabla \phi \rightarrow(U, 0,0)$ as $x^{2}+y^{2}+z^{2} \rightarrow \infty$

On the free surface the kinematic and the dynamic conditions must be satisfied as stated in the following equations

$$
\begin{aligned}
& \phi_{x} \eta_{x}+\phi_{x} \eta_{x}-\phi_{z}=0 \quad \text { on } \quad z=\eta \\
& \eta=\frac{1}{2 g}\left(U^{2}-\nabla \phi \cdot \nabla \phi\right)
\end{aligned}
$$

Since Eqs. (11) and (12) are fully nonlinear equations, in this paper the iteration procedure is used to solve the free surface problem based on the Rankine source panel method [9] and the panel cutting method are applied to achieve the wave-making resistance as the objective function [1].

Having obtained the velocity potential and hence the flow velocity, the pressure coefficient at each panel can be found using Bernoulli's equation

$$
C_{p}=1-\frac{\nabla \phi \cdot \nabla \phi}{U^{2}}-2 \frac{z}{F n^{2}}
$$

The wave-making resistance coefficient $\mathrm{C}_{\mathrm{W}}$ is hence given by the pressure integral over the wetted hull surface.

$C_{W}=-\frac{\int_{S} C_{p} n_{x} d s}{S}$

The frictional resistance coefficient is given by the ITTC 1957 model-ship correlation line formula.

$$
C_{F}=\frac{0.075}{\left(\log _{10} R n-2\right)^{2}}
$$

where $S$ is the wetted surface of the hull and local Reynolds number is used for each element, based on the local velocity.

Finally the resistance coefficient is obtained as follows

$$
C_{T}=C_{W}+(1+k) C_{F}
$$

where the form factor $(1+k)$ is calculated using the empirical formula presented by Holtrop et. al. [4].

\section{Modification of Hull Geometry}

\subsection{B-Spline Surface Modeling}

In the implementation of an optimization procedure, it is essential to have an efficient algorithm for geometry modification. The modified geometry should meet original design requirements without discontinuities in the surface and should be generally as smooth as possible. For ship hull optimization, it is necessary to use factors which are related to hull geometry generation as design variables. In this case, the increase of design variables means the increase of computation time. If the number of design variables is too small, it might be difficult to get realistic hull shape. These requirements have been obtained by using a B-spline patch:

$$
Q(u, v)=\sum_{i=1}^{n_{i}+1} \sum_{j=1}^{n_{j}+1} B_{i, j} N_{i, k}(u) M_{j, l}(v)
$$

where $B_{i, j}$ s are the control vertices of a defining polygon net, $N_{i, k}(u)$ and $M_{j, l}(v)$ are the Bspline basis function in the bi-parametric $u$ and $v$ directions, respectively. The definition for the basis functions is as follows

$$
\begin{aligned}
& N_{i, 1}(u)=\left\{\begin{array}{cc}
1 & \text { if } x_{i} \leq u<x_{i+1} \\
0 & \text { otherwise }
\end{array}\right. \\
& N_{i, k}(u)=\frac{\left(u-x_{i}\right) N_{i, k-1}(u)}{x_{i+k-1}-x_{i}}+\frac{\left(x_{i+k}-u\right) N_{i+1, k-1}(u)}{x_{i+k}-x_{i+1}}
\end{aligned}
$$

and 


$$
\begin{aligned}
& M_{j, 1}(v)= \begin{cases}1 & \text { if } y_{j} \leq v<y_{j+1} \\
0 & \text { otherwise }\end{cases} \\
& M_{j, l}(v)=\frac{\left(v-y_{j}\right) M_{j, l-1}(v)}{y_{j+l-1}-y_{j}}+\frac{\left(y_{j+l}-v\right) M_{j+1, l-1}(v)}{y_{j+l}-y_{j+1}}
\end{aligned}
$$

where the $x_{i}$ and $y_{j}$ are elements of knot vectors.

\subsection{B-spline Surface Fitting}

When the surface is described by external data, it is convenient to obtain an initial non-flat B-spline surface approximating the hull for subsequent real time interactive modification. This requires determining the defining polygon net from an existing network of three dimensional surface data points.

For each known surface data point Eq. (17) provides a linear equation in the unknown $B_{i, j}$ 's and similarly for all the surface data points. In matrix notation this can be written as

$[D]=[C][B]$

where $C_{i, j}=N_{i, k} M_{j, l}$. For arbitrary $r * s$ topologically rectangular surface point data, $[D]$ is an $r^{*} s \times 3$ matrix containing the threedimensional coordinates of the surface point data, [C] is an $r^{*} s \times n^{*} m$ matrix of the products of the $\mathrm{B}$-spline basis functions, and $[B]$ is an $n * m \times 3$ matrix of the three-dimensional coordinates of the required polygon net points.

Since for any arbitrary $r^{*} s$ topologically rectangular surface point data, $[C]$ is not normally square, a solution can only be obtained in some mean sense. In particular

$$
[B]=\left[[C]^{T}[C]\right]^{-1}[C]^{T}[D]
$$

The $u$ and $v$ parametric values for each surface data are obtained using a chord length approximation $[5,10]$.

\section{Application}

The method described above was applied to an op- timization problem in which numerical computation had been performed using the Series 60 hull $\left(\mathrm{C}_{\mathrm{B}}=0.60\right)$ as a parent hull at the design speed $(\mathrm{Fn}=0.316)$.

\subsection{Design Variables}

Since the potential flow solver was used to calculate the wave-making resistance as an objective function, the panels which define the shape of the ship surface should be generated at each iteration step during the whole optimization process. To make the optimization algorithm more stable for the numerical computation the representation of the ship needed to be sufficiently flexible and robust to permit any type of hull form modifications, while involving only a moderate number of unknowns was an important factor of hull-form optimization because of the more trivial reason of the severe time constraints that are the rule in practical ship design.

As shown in Fig. 1 the ship surface was divided into 2 zones and the ship optimization was only performed to the region $25 \%$ from the bow in which the flow was assumed to be the potential flow. Each zone was defined by the B-spline surface patch. In case of the optimization region the surface patch with 10 by 7 control net ( 7 control points for each 10 section) was used to represent a hull form and the control vertices were used as design variables.

The design variables were repositioned according the optimization algorithm and used to generate the computation panel for numerical analysis. For the other zone the control vertices were generated but not modified during the optimization process.

Fig. 2 shows the control vertices which were allowed to move in $\mathrm{x}$-direction. The control vertices at the first and second sections had the same $\mathrm{x}$ coordinates at each row to enforce continuity of curvature across the centerplane.

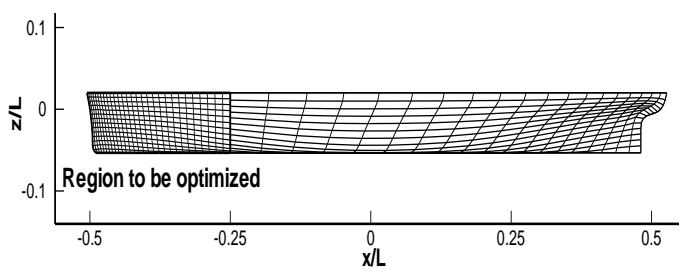

Fig. 1 Region to be optimized 


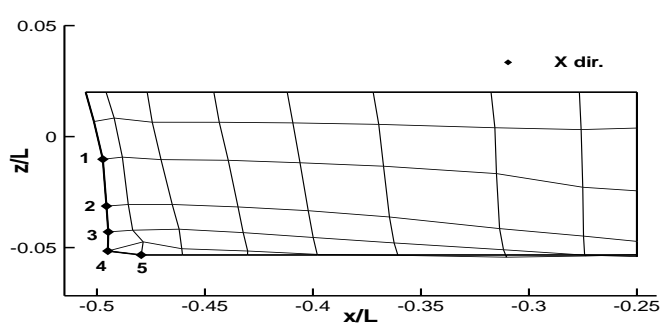

Fig. 2 Design variables varying in $\mathrm{x}$ direction

As the control vertices moved in $\mathrm{x}$-direction the $\mathrm{x}$ coordinates of the other control vertices between the second and last sections moved inversely proportional to the distance between the two points. To maintain the reasonable hull form shape the range of the movement of the control vertices was just confined as shown in Eq. (24).

$$
\begin{aligned}
-0.5500<x_{1}<-0.4972 \\
-0.5500<x_{2}<-0.4955 \\
-0.5500<x_{3}<-0.4947 \\
-0.5500<x_{4}<-0.4949 \\
-0.5500<x_{5}<-0.4749
\end{aligned}
$$

Fig. 3 shows the design variables which were allowed to move in y-direction. To maintain the reasonable ship shape the range of the movement of the control vertices was also confined as shown in Eq. (25).

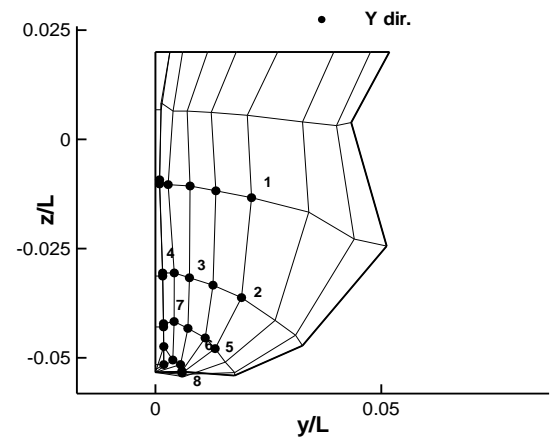

Fig.3 Design variables varying in y direction

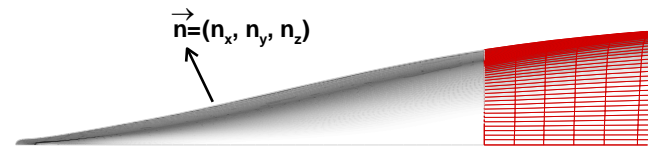

Fig.4 Definition of $n$

$$
\begin{aligned}
& 0.0212<y_{1} / L<0.0300 \\
& 0.0190<y_{2} / L<0.0300 \\
& 0.0000<y_{3} / L<0.0160 \\
& 0.0000<y_{4} / L<0.0100 \\
& 0.0132<y_{5} / L<0.0300 \\
& 0.0110<y_{6} / L<0.0300 \\
& 0.0000<y_{7} / L<0.0140 \\
& 0.0058<y_{8} / L<0.0060
\end{aligned}
$$

The range of the movement for the other design variables in $\mathrm{y}$-direction was confined as follows

$0.0000<y / L<0.0300$

\subsection{Constraint Conditions}

The Constraints of the displacement and the wetted surface area of the ship were pre-described depending on the requirement of ship designer since they were most important factors in ship design.

In the present study, the constraint for the displacement was set as follows, which might be a severe penalty from a resistance point of view.

$\Delta \geq \Delta_{\text {initial }}$

The constraint for the normal vector to the hull surface was applied to avoid the evolution of the hull to an undesirable direction.

$n_{x} \leq 0$

$n_{y} \geq 0$

Eqs. (28) and (29) were important constraints since all numerical computations during the optimization process were performed automatically so that the ship had a reasonable hull shape without external modification. During the optimization procedure it was found that the numerical computations could be more stable and the ship shape could be modified in a smoother way by enforcing them.

\subsection{Computational Results}




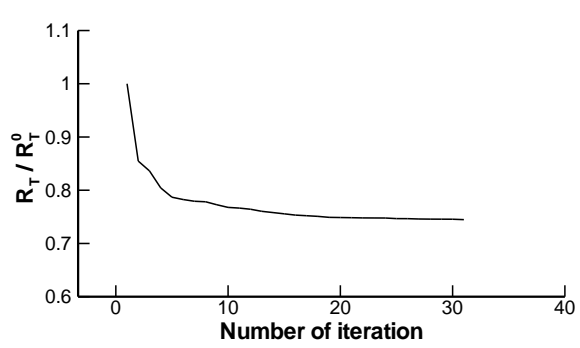

Fig. 5 Convergence history

Fig. 5 shows the variation of $R_{T} / R_{T}^{0}$ with respect to the number of iteration.

Here, $R_{T}^{0}$ was the resistance for the initial hull form. Fig. 5 shows that the resistance was quickly reduced during first 5 iterations and after 5 iteration the reduction rate was slowly decreased and finally converged.

As shown in Fig. 6, the volume of the middle part of the bow is increased dramatically and the bow is bulb-shaped compared with the parent hull. Although some constraints were provided to prevent an undesirable hull form generation, it is still noticed that there are some unusual curves which seem to be not easily acceptable from a practical point of view and the optimized hull was ,therefore, slightly modified manually using a surface fitting technique. Fig. 7 shows a comparison between the optimized hull and the final version of the optimized hull with slight modification and a great care had been taken to maintain the main feature of the optimized hull.
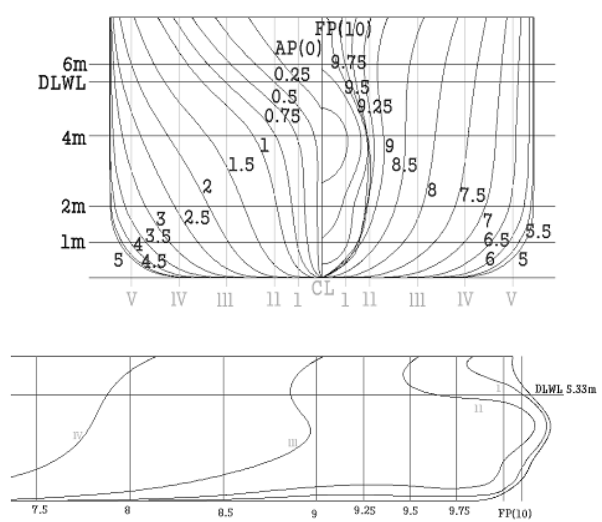

Fig. 6 The body plan and the buttock line for the optimized hull
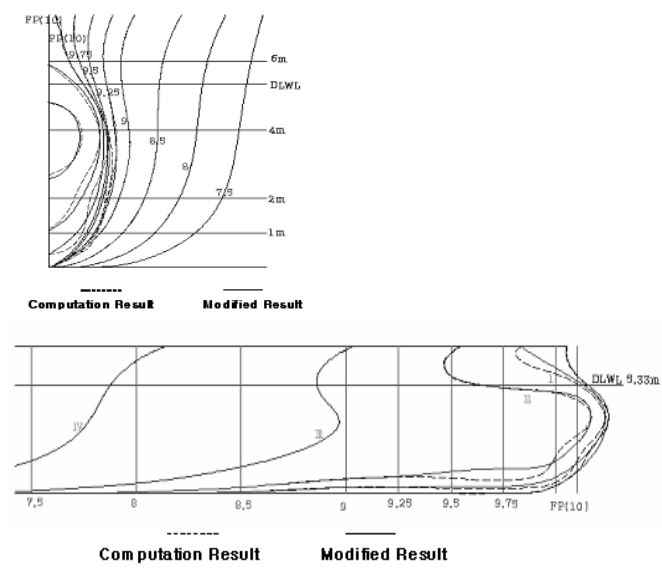

Fig. 7 The optimized hull and the modified optimized hull

Fig. 8 and 9 compared the body plans and the waterlines of the parent hull and the optimized hull and the sectional area curves were compared in Fig. 10. The optimized hull has a big bulb compared with the parent hull.

In Fig. 11 the wave profiles in longitudinal direction in the vicinity of the hull were compared and the measured wave profile of the parent hull was also compared with the computed results. For the parent hull the computed results predicted slightly bigger wave heights than the measured data near the stern part. It is because the potential flow solver ignores the viscosity of the fluid. The wave height in the bow part of the optimized hull was considerably reduced mainly due to the existence of the bulb. Over the free surface area the wave height was reduced as shown in Fig. 12.

\section{Parent Hull Optimized Hull}

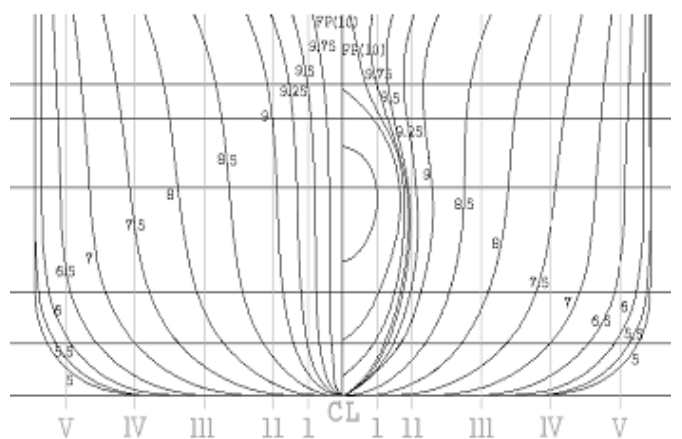

Fig. 8 Comparison of the body plans 

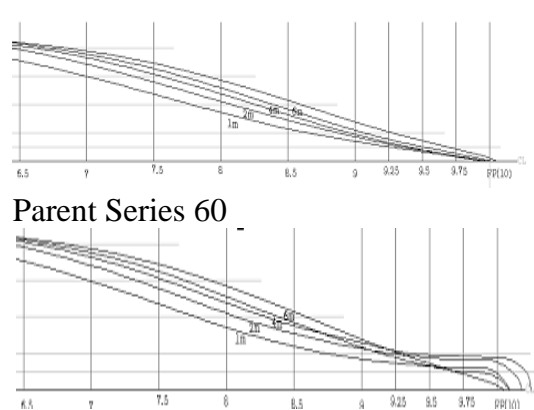

Optimized Series 60

Fig. 9 Comparisons of the waterlines

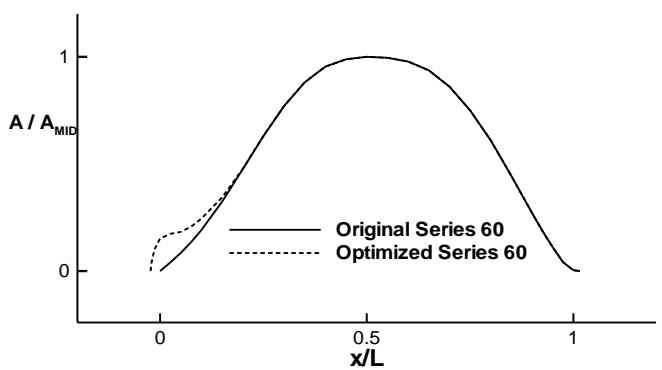

Fig. 10 Comparison of the sectional area curves

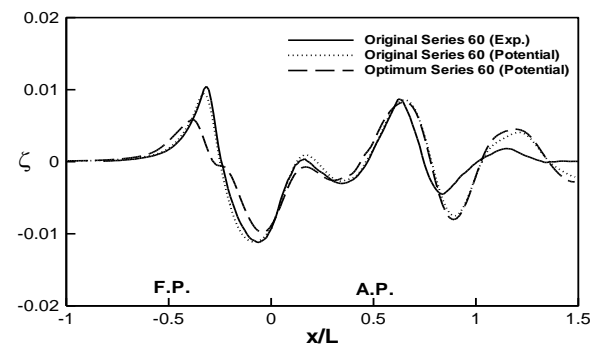

Fig.11 Comparison of the wave profiles at $\mathrm{y} / \mathrm{Lpp}=0.08$ $(\mathrm{Fn}=0.316)$

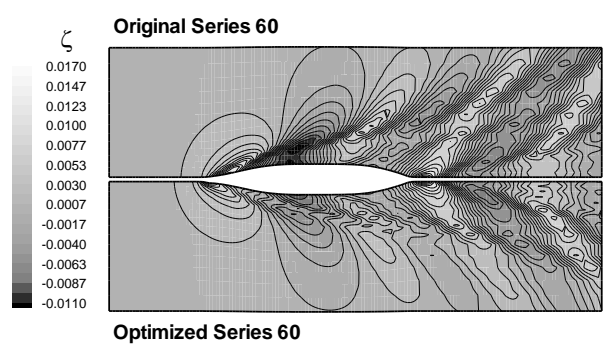

Fig. 12 Comparison of the wave contours $(\mathrm{Fn}=0.316)$

\subsection{Experimental Validation}

The model test was carried out for the parent hull and the optimized hull. Fig. 13 shows the models of the parent hull and the optimized hull. The principal parameters of the series $60\left(\mathrm{C}_{\mathrm{B}}=0.60\right)$ hull as a parent hull are shown in Table 1 .

As shown in Fig. 14 and in Table 2, the resistance was reduced by $13.3 \%$ compared with the parent hull at the design speed and the wetted area and total volume of the optimized hull were increased by $0.9 \%$ and $2 \%$. In Fig. 15 the residuary resistance of the optimized hull was reduced by $39.1 \%$ while the computational result shows $26.5 \%$ reduction, where the residual resistance was calculated by the resistance minus the frictional resistance which was calculated from the ITTC 1957 model-ship correlation line.

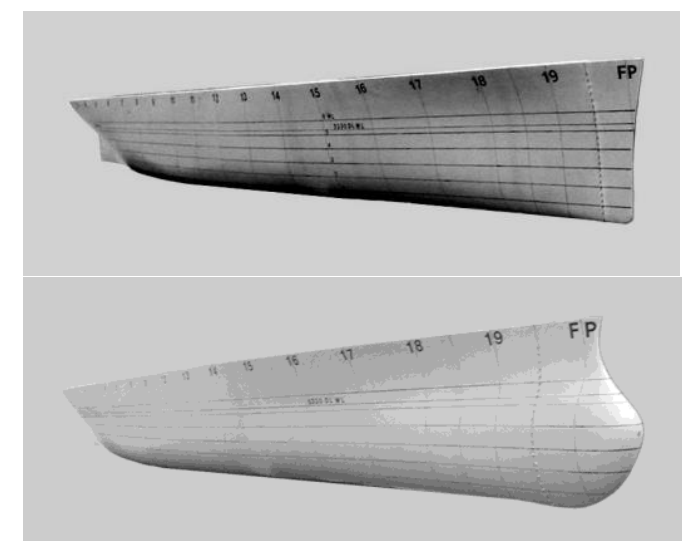

Fig. 13 Models (upper figure represents the parent hull; lower figure represents the optimized hull)

Table 1 Pricipal parameters of the series 60 hull

\begin{tabular}{l|l|l|l}
\hline $\begin{array}{l}\text { Length } \\
(\mathrm{m})\end{array}$ & $\begin{array}{l}\text { Breadth } \\
(\mathrm{m})\end{array}$ & $\begin{array}{l}\text { Draft } \\
(\mathrm{m})\end{array}$ & $\begin{array}{l}\text { Block } \\
\text { coefficient }\end{array}$ \\
\hline 3.5 & 0.467 & 0.187 & 0.60 \\
\hline
\end{tabular}

Table 2 hydrostatic/dynamic data for the parent hull and the optimized hull at design speed $(\mathrm{Fn}=0.316)$

\begin{tabular}{|c|c|c|c|c|}
\hline & & Parent & Optimized & $\Delta(\%)$ \\
\hline & $\left(\mathrm{m}^{3}\right)$ & 0.115 & 0.117 & 2.0 \\
\hline & $S_{\text {WET }}\left(\mathrm{m}^{2}\right)$ & 1.539 & 1.553 & 0.9 \\
\hline \multirow{2}{*}{ Num. } & $\mathrm{C}_{\mathrm{W}} \quad\left(* 10^{3}\right)$ & 1.860 & 1.360 & -27.1 \\
\hline & $\mathrm{R}_{\mathrm{W}} \quad(\mathrm{N})$ & 0.156 & 0.115 & -26.5 \\
\hline \multirow{3}{*}{ Exp. } & $\mathrm{C}_{\mathrm{R}} \quad\left(* 10^{3}\right)$ & 2.040 & 1.220 & -40.5 \\
\hline & $\mathrm{R}_{\mathrm{RM}} \quad(\mathrm{N})$ & 4.620 & 2.820 & -39.1 \\
\hline & $\mathrm{R}_{\mathrm{TM}}(\mathrm{N})$ & 12.48 & 10.83 & -13.3 \\
\hline
\end{tabular}






Fig. 14 Comparison of the resistances $\left(\mathrm{R}_{\mathrm{T}}\right)$

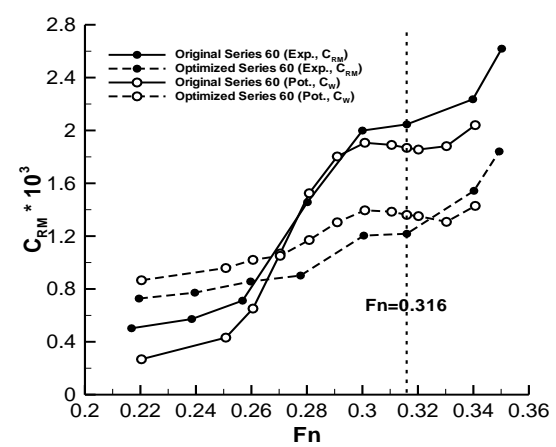

Fig.15 Comparison of the residual/wave-making resistance coefficients $\left(\mathrm{C}_{\mathrm{RM}} / \mathrm{C}_{\mathrm{W}}\right)$

\section{Conclusion}

The hull optimization algorithm was applied to the series $60\left(C_{B}=0.60\right)$ hull as a parent hull and generated the optimized hull form from the minimum resistance point of view. The model tests were performed to validate the applied algorithm and the computed results were compared with the experimental results.

Experimental results showed the optimized hull gave $13 \%$ reduction in the resistance and $40 \%$ reduction in the residuary resistance. It should be, however, noticed that the reduction rate of the resistance mostly depends on choosing a parent hull. The results, however, indicated that the proposed optimized algorithm and the constraint conditions might be effectively applied to design a better hull form.

\section{References}

[1] Choi, H. J., Chun, H. H., Park, I. L. and Kim, J., Panel cutting method: new approach to generate panels on a hull in Rankine source potential approximation, International Journal of Naval Architecture and Ocean Engineering, Vol. 3, pp.
225-232 (2011).

[2] Harries, S., Abt, C. and Heimann, J., From Redesign to optimal hull Line by means of Parametric Modeling, The Institute of Naval Architecture and Ocean Engineering of the Technical University Berlin \& FRIENDSHIPSystems, COMPIT2003 (2003).

[3] Hino, T., Kodama, Y., and Hirata, N., Hydrodynamic Shape Optimization of Ship Hull Forms Using CFD, Proceedings 3rd Osaka Colloquium on Advanced CFD Applications to Ship Flow and Hull Form Design, Osaka, Japan, May 25-27, pp. 332-340 (1998).

[4] Holtrop, I. J., A Statistical Re-analysis of Resistance and Propulsion Data, International Ship-Building Progress, Vol. 31, pp. 272-276, (1984).

[5] Huang, C. H., Chiang, C. C. and Chou, S. K., An Inverse Geometry Design Problem in Optimizing Hull Surfaces, Journal of Ship Research, Vol. 42, No. 20, pp. 79-85, (1998).

[6] Lowe, T. W. and Steel, J., Conceptual Hull Design Using a Genetic Algorithm, Journal of Ship Research, Vol. 47, No. 3, pp. 222-236 (2003).

[7] Markov, N. E. and Suzuki, K., Hull form optimization by shift and deformation of ship sections, Journal of Ship Research, Vol. 45, No. 3, pp 197-204, (2001).

[8] Peri, D., Rosetti, M. and Campana E. F., Design Optimization of Ship Hulls via CFD Techniques, Journal of Ship Research, Vol. 45, No. 2, pp. 140-149 (2001).

[9] Raven, H. C., A Practical Nonlinear Method for Calculating Ship Wavemaking and Wave Resistance, Proc. 19th ONR Symposium on Naval Hydrodynamics, Seoul (1992).

[10]Rogers, D. F. and Adams J. A., Mathematical Elements for Computer Graphics, McGrawHill, New York (1990).

[11]Suzuki, K., Kai, H., Saha, G. K., Hydrodynamic optimization of ship hull forms in shallow water, Journal of Marine Science and Technology (2004).

[12]Tahara, Y., Himeno, Y. and Tsukahara, T., An Application of Computational Fluid Dynamics to Tanker Hull Form Optimization Problem, Proceedings 3rd Osaka Colloquium on Advanced CFD Applications to Ship Flow And Hull Form Design, Osaka, Japan, pp. 515-531 
(1998).

[13]Tahara, Y., Stern, F., Himeno, Y., Computational Fluid Dynamics-Based Optimization of Surface Combatant, Journal of Ship Research, Vol. 28, No. 4, pp. 273-287 (2004).

[14]Vanderplaats, G. N., Numerical Optimization Techniques for Engineering Designs, McGrowHill, New York (1984).

[15]Zhang, B. J., Research on optimization of hull lines for minimum resistance based on Rankine source method, Journal of Marine Science and Technology, Vol. 20, No. 1, pp. 89-94 (2012).

[16]Zhang, B. J., The optimization of the hull form with the minimum wave making resistance based on Rankine source method, Journal of Hydrodynamics, Vol. 21, No. 2, pp. 277-284 (2009). 